\title{
Minimally Invasive Multilevel Percutaneous Pedicle Screw Fixation for Lumbar Spinal Diseases
}

\author{
Seong Son, Sang Gu Lee, Chan Woo Park, Woo Kyung Kim \\ Department of Neurosurgery, Gachon University, Gil Hospital, Incheon, Republic of Korea
}

\begin{abstract}
Objective: There are rare reports on the result of multilevel ( $\geq 3$ levels) percutaneous pedicle screw fixation (PPF). The purpose of this study was to report the clinical experiences for multilevel PPF of the lumbar spine.

Methods: A total of 17 patients of lumbar spinal disease ( 7 degenerative diseases, 6 infectious diseases, and 4 traumatic instabilities) underwent neural decompression and multilevel PPF. There were 8 men and 9 women with a mean age of 61.4 years (range, 25-84) and a mean follow-up period of 23.2 months (range, 13-48). The average PPF level was 3.7. A retrospective review of clinical, radiological, and surgical data was conducted.

Results: "Excellent" or "good" clinical results were obtained in 15 patients (88.2\%) according to the Odom's criteria. The average improvement of visual analogue scale was 5.2 points (from 9.3 to 4.1 ), and the average improvement of Oswestry Disability Index was 36.2 (from 71.2 to 35.0 ) at the last visit $(p<0.05)$. The fusion rate was $88.2 \%$, but, screw loosening was occurred in 2 patients, and adjacent segmental degeneration was occurred in 2 patients. There was no statistical significance in the change of total lumbar lordotic angle. The average operation time was 5.9 hours, with an estimated blood loss of 550 $\mathrm{ml}$ and bed rest duration of 2.0 days.

Conclusion: Although the current study examined a small sample with relatively short term follow up periods, our study results demonstrate that multilevel PPF is feasible and safe for selective lumbar spinal diseases.
\end{abstract}

Key Words: Spinal fusion $\cdot$ Lumbar vertebrae $\cdot$ Bone screw

\section{INTRODUCTION}

Minimally invasive spine surgery may allow for surgery of the lumbar spine with considerably less blood loss and soft tissue damage. Recently, to reduce the adverse effect of the open technique of pedicle screw, the percutaneous pedicle screw fixation (PPF) is becoming more widespread in spinal surgery. Many studies about PPF have been reported, but still, there are rare reports about the result of multilevel ( $\geq 3$ levels) $\mathrm{PPF}^{4,10,12,18)}$. This study analyzed the clinical, radiological, and surgical results of the multilevel PPF in various lumbar spinal diseases.

\section{MATERIALS AND METHODS}

\section{Patient population}

From March 2008 to April 2011, a total of 17 patients of

- Received: November 12, 2012 • Revised: December 18, 2012

- Accepted: December 24, 2012

Corresponding Author: Sang Gu Lee, MD, PhD

Department of Neurosurgery, Gil Hospital, 1198 block, Guweol-Dong,

Namdong-Gu, Incheon 405-220, Republic of Korea

Tel: +82-32-460-3304, Fax: +82-32-460-3899

Email: samddal@gilhospital.com lumbar spinal disease underwent neural decompression, interbody fusion and multilevel PPF by one neurosurgeon. There were 8 men and 9 women with a mean age of 61.4 years (range, 2584) and a mean follow-up period of 23.2 months (range, 18-43).

Below is a group list of our indications for the procedure.

1) Multilevel degenerative diseases such as spinal stenosis, spondylolisthesis (Grade I), or instability with symptoms refractory to conservative treatment.

2) Severe bone destruction with a spinal cord compression or a nerve root compression due to infectious diseases such as tuberculous spondylitis or pyogenic spondylitis.

3) Spinal instability with a spinal cord compression or a nerve root compression due to trauma.

Out of 17 patients, 7 patients were degenerative diseases (5 spinal stenosis with instability, 2 spondylolisthesis), 6 patients were infectious diseases (4 pyogenic spondylitis, 2 tuberculous spondylitis) and 4 patients were burst fractures with thecal sac compression and instability (Table 1). The patients, with excessive spondylolisthesis or severe spinal deformity such as scoliosis, kyphosis, and rotational deformity, were excluded from PPF procedure.

\section{Procedures and instruments}

We performed neural decompression and interbody fusion 
Table 1. Summary of patients' data patients' data

\begin{tabular}{|c|c|c|c|c|c|c|}
\hline & Case & Sex & Age & Diagnosis & Decompression (levels) & PPF levels \\
\hline \multirow{7}{*}{$\begin{array}{l}\text { Degenerative } \\
\text { disease }\end{array}$} & 1 & $\mathrm{~F}$ & 70 & Stenosis with instability L2-5 & $\mathrm{LN}^{*}(1), \mathrm{PLIF}^{+}(2)$ & 3 \\
\hline & 2 & $M$ & 61 & Stenosis with instability L1-4 & $\operatorname{LN}(1), \operatorname{PLIF}(1)$ & 3 \\
\hline & 3 & $\mathrm{~F}$ & 61 & Stenosis with instability L2-5 & $\operatorname{LN}(2), \operatorname{PLIF}(1)$ & 4 \\
\hline & 4 & M & 53 & Stenosis with instability L2-5 & $\operatorname{LN}(1), \operatorname{pLIF}(2)$ & 3 \\
\hline & 5 & $\mathrm{~F}$ & 69 & Stenosis with instability L3-S1 & LN (1), PLIF 2 & 3 \\
\hline & 6 & $\mathrm{~F}$ & 55 & Stenosis and spondylolisthesis L2-5 & PLIF (3) & 3 \\
\hline & 7 & M & 84 & Stenosis and spondylolisthesis L2-5 & PLIF (3) & 5 \\
\hline \multirow{6}{*}{$\begin{array}{l}\text { Infectious } \\
\text { disease }\end{array}$} & 8 & M & 70 & Pyogenic spondylitis L2-3 & $\operatorname{LN}(1), \operatorname{PLIF}(2)$ & 4 \\
\hline & 9 & $M$ & 71 & Pyogenic spondylitis L3-5 & Ant $\mathrm{Co}^{*}(3)$ & 5 \\
\hline & 10 & M & 25 & Tuberculous spondylitis L2-3 & Ant Co (2) & 3 \\
\hline & 11 & $\mathrm{~F}$ & 76 & Tuberculous spondylitis T12-L1 & Post Cu (3) & 6 \\
\hline & 12 & M & 69 & Pyogenic spondylitis L1-2 & Post Cu (2) & 3 \\
\hline & 13 & M & 49 & Pyogenic spondylitis $L 4-5$ & $\operatorname{PLIF}(1)$ & 3 \\
\hline \multirow{4}{*}{$\begin{array}{l}\text { Traumatic } \\
\text { instability }\end{array}$} & 14 & $\mathrm{~F}$ & 67 & Instability with cord injury T12-L2 & $\mathrm{LN}(2)$, Post $\mathrm{Cu}^{\S}(2)$ & 5 \\
\hline & 15 & $\mathrm{~F}$ & 57 & Instability with cord injury L1-2 & Ant Co (2) & 4 \\
\hline & 16 & $\mathrm{~F}$ & 60 & Instability with cord injury L1-2 & LN (1), Post Cu (1) & 3 \\
\hline & 17 & $\mathrm{~F}$ & 47 & Instability with cord injury T12-L1 & LN (1), Post Cu (1) & 3 \\
\hline Mean & & & 61.4 & & & 3.7 \\
\hline
\end{tabular}

first. The posterior lumbar terbody fusion (PLIF) with facectectomy via midline incision was performed in 9 patients, the posterior lumbar currettage and interbody fusion in $5 \mathrm{pa}$ tients, and the anterior lumbar corpectomy and interbody fusion in 3 patients. Allograft iliac bone, mesh cage containing allograft bone chips, or polyetheretherketone (PEEK) cage containing allograft bone chips were used in interbody fusion.

And then, multilevel PPF was performed in prone position using CD Horizon ${ }^{\circledR}$ Longitude $^{\circledR}$ (Medtronic Sofamor Danek, Memphis, TN,USA), CD Horizon ${ }^{\circledR}$ Sextant ${ }^{\circledR}$ II (Medtronic Sofamor Danek, Memphis, TN, USA), Viper ${ }^{\circledR}$ II (DePuy Spine, Raynham, MA, USA), or AnyPlus ${ }^{\circledR}$ (GS Spine, Santa Cruz, CA, USA). The screws were placed percutaneously using fluoroscopic guidance. The cannulated screws, which were inserted over a guide wire, had extenders attached to them, which had a slot to receive the rod. Since the entry point of screw insertion in the middle pedicle is most important for alignment of spine, the insertion of screw in the middle pedicle was carried out lastly.

The slot was large enough in the unreduced position to accept a rod that was passed again percutaneously. The rod is contoured according to the sagittal contour desired and then passed free hand through the slots under direct fluoroscopic control. Once the rod is appropriately positioned through all the screw extender slots, the extender is reduced to seat the rod into the tulip of the screw head. Once reduced, the top locking nut is inserted to fix the rod to the screw starting from the caudal screw and working proximally in sequential fashion. Once all the nuts are in place, the extender is unseated and detached from the screw. Compression or distraction can be applied to the extenders as desired, to gain further correction.

The average PPF level was 3.7 (range, 3-6).

\section{Outcome parameters}

A retrospective review of clinical, radiological, and surgical data was conducted.

Of the back pain or leg pain, the severity of main symptom was recorded using visual analog scale (VAS). Functional outcomes were measured using the Oswestry Disability Index (ODI) scores, and patient's satisfactions were recorded using Odom's criteria during follow-up period.

For radiological evaluation, we examined the dynamic Xrays, magnetic resonance imaging (MRI), and computed tomography (CT) prior to surgery, and also, the dynamic X-rays and CT consecutively during follow-up period.

The accuracy of screw position was analyzed by immediate postoperative CT. In CT scan, screw perforation of any aspects of cortex was checked, and when all parts that constitute the 
screw were located in pedicle and vertebral cortex, it was considered as screw accurately inserted.

To evaluate sagittal alignment, total lumbar lordotic angle (TLA) was measured on lateral radiograph in neutral position, and adjacent segmental angle (ASA) was measured on flexionextension view (Fig. 1). We defined definite change of ASA on the standing lateral film as sagittal translation of the adjacent vertebral body above fused level greater than $3 \mathrm{~mm}$ and/or ASA greater than 10 degrees ${ }^{19}$. TLA and ASA were checked at the preoperative period, 1 month after the operation, 6 months after the operation, and the last follow-up.

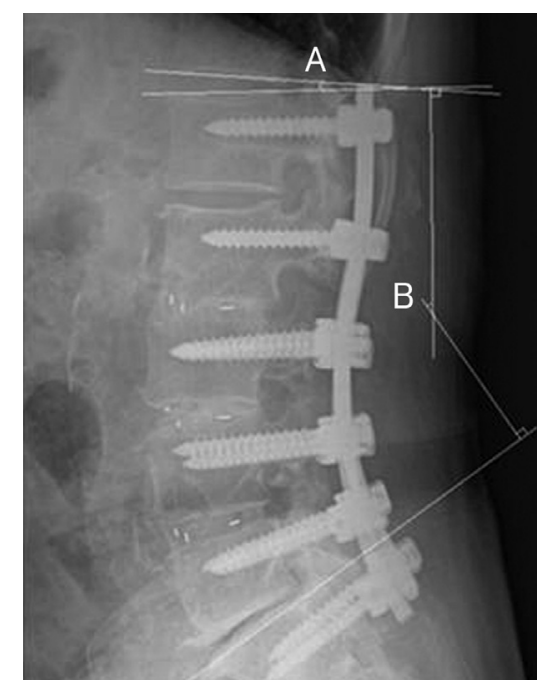

Fig. 1. The sagittal angle measurement by Cobb's angle method on plain X-ray. The adjacent segmental angle (ASA) was measured as total sum of intervertebral angles between flexion and extension lateral radiographs (A). To measure it, line along the end plate of adjacent vertebra body and line along the end plate of the most superior or inferior fused vertebra body were drawn. The total lumbar lordotic angle (TLA) was defined as the angle subtended by the superior end plate line of $\mathrm{L} 1$ and the superior end plate line of S1 (B).
The bony fusion rate of interbody fusion and screw failure such as fracture or loosening were evaluated by dynamic Xrays and CT during follow-up period. The degree of bone fusion was based on the classification of Brantigan and Steffee ${ }^{2)}$ (Table 2), and we regarded Grade 4 or 5 as a state of bone fusion. The screw loosening was confirmed when we observed more than $1 \mathrm{~mm}$ thick radiolucent zone (halo sign) around screw on plain radiographs. Also, we have identified the development of the late postoperative complications such as instability and instrument failure.

Surgical outcomes were evaluated by checking the operation time, estimated blood loss (EBL), duration of postoperative bed rest and length of hospital stay. The occurrence of perioperative morbidities such as neurologic deterioration, cerebrospinal fluid (CSF) leakage, wound infection, pneumonia, heart problem, urinary difficulty, epidural hematoma, and deep vein thrombosis were checked. Also, we tracked the frequency of reoperation.

\section{Statistical methods}

For statistical analysis, paired samples $t$ test was conducted using SPSS software (version 17.0, SPSS Inc, Chicago, IL, USA). A probability value of less than 0.05 was considered significant.

\section{RESULTS}

\section{Clinical outcomes}

The mean VAS score at the 1 month after the surgery 6.7 (range, 5-9) was significantly lower than the preoperative score, 9.3 (range, 9-10). The mean VAS score decreased at each followup evaluation and was significantly lower at the last follow-up 4.1 (range, 2-5) compared with the preoperative score (Fig. 2). The mean ODI score also improved from 71.2 (range, 67-

Table 2. Description of fusion result by Brantigan and Steffee

Grade 1 Obvious collapse of construct due to pseudoarthrosis, loss of disc height, vertebral slip, broken screws, displacement of the cage, resorption of bone graft

Grade 2 Probable significant resorption of the bone graft due to pseudoarthrosis, major lucency, or gap visible in fusion area (2 mm around the entire periphery of graft)

Grade 3 Uncertain non-union, bone graft visible in the fusion area at approximately the density originally achieved at surgery. A small lucency or gap may be visible involving a portion of the fusion area with at least half of the graft area.

Grade 4 Probable fusion bone bridges entire fusion area with at least the density achieved at surgery. There should be no lucency between the donor and vertebral bone.

Grade 5 Fusion bone in the fusion area is radiographically more dense and mature than originally achieved by surgery. Optimally, there is no interface between the donor bone and the vertebral bone, although a sclerotic line between the fusion area, resorption of the anterior traction spur, anterior progression of the graft within disc space, and fusion of facet joints. 


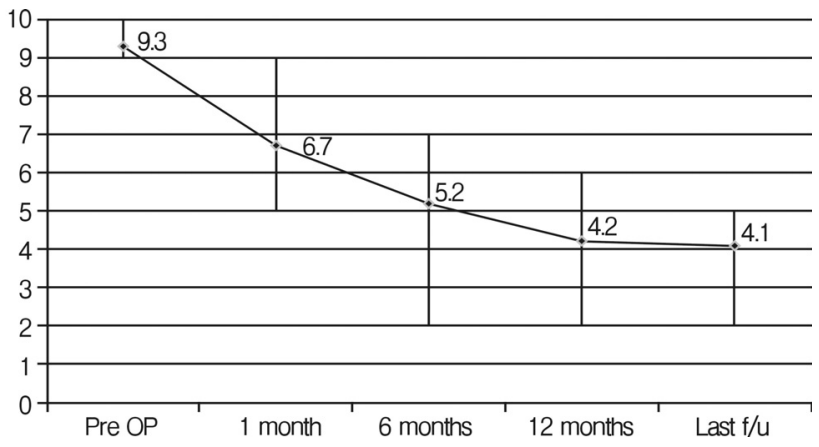

Fig. 2. Graph showing visual anolog scale (VAS) before the surgery and during the follow-up period. The mean improvement of VAS from the pre-operation to the last follow-up was 5.2 points (from 9.3 to 4.1) $(p<0.05)$. Months 1, 6, 12 and the last follow- up are represented on the $X$ axis. The $Y$ axis represents the score.

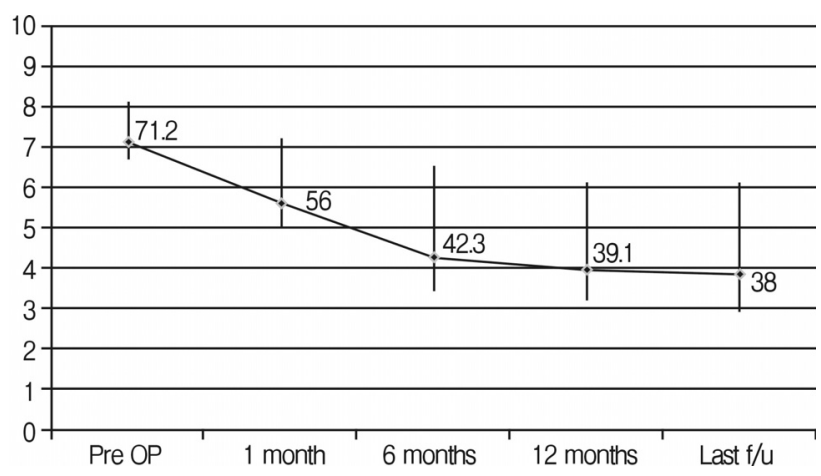

Fig. 3. Graph showing Oswestry Disability Index (ODI) before the surgery and during the follow-up period. The mean improvement of ODI from the pre-operation to the last follow-up was 36.2 (from 71.2 to 35.0) $(p<0.05)$. Months 1, 6, 12 and the last follow-up are represented on the $X$ axis. The $Y$ axis represents the score.

81) preoperatively to 38.0 (range, 29-61) at the last follow-up (Fig. 3). Both VAS and ODI score improved after the surgery and the improvement maintained during the follow-up period with statistical significance $(\mathrm{p}<0.05)$.

According to the Odom's criteria, the results were excellent in 6 patients (35.3\%), good in 7 patients (41.2\%), and fair in 4 patients (23.5\%) at the 6-month follow-up, and excellent in 7 patients (41.2\%), good in 8 patients (47.0\%), and fair in 2 patients $(11.8 \%)$ at the last follow-up. Therefore, the clinical success rate according to the Odom's criteria was $88.2 \%$ (Fig. 4).

\section{Radiological outcomes}

In all the patients, by CT scan immediately after the surgery, the cases of screw malposition were 6 (4.1\%) out of 146 screws.

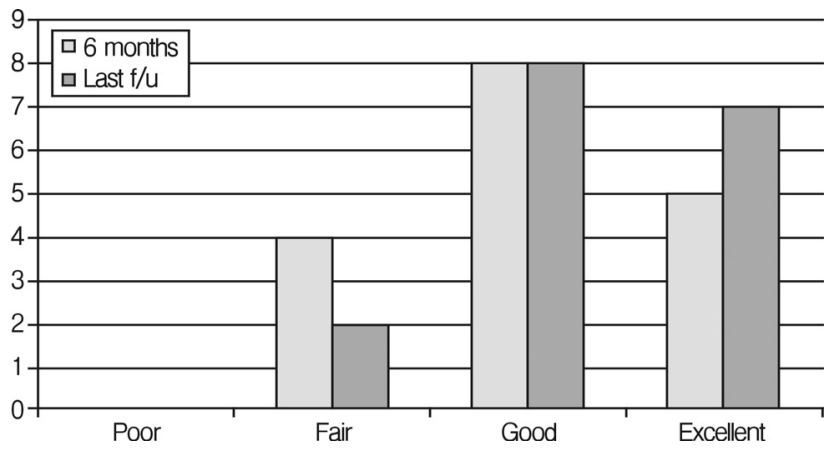

Fig. 4. The number of patients according to Odom's criteria at the 6 months after surgery and at the last follow-up. The $Y$ axis represents the number of patients.

However, there was no occurrence of neurological deficit or vascular complications, and also no need of screw correction.

The mean TLA increased from $34.7^{\circ}$ before the surgery to $38.0^{\circ}$ at the 1 month after the surgery, however, decreased to $35.9^{\circ}$ at the last follow-up. The mean ASA was $5.7^{\circ}$ at preoperation and $7.0^{\circ}$ at the last follow-up. The mean ASA of the caudal adjacent segment was $5.6^{\circ}$ at pre-operation and 5.3 at the last follow-up. There was no statistical significance in the changes of the TLA and ASA. Only 2 patients (11.8\%) showed definite change of ASA of cranial adjacent segment, and all of them were asymptomatic.

During follow-up period, there were bony fusions in 15 patients out of 17 patients who underwent interbody fusion, and thus fusion rate was $88.2 \%$. On the other hand, there were screw loosening in 2 patients, but there was no pull-out or fracture of screw. Among 2 patients of screw loosening, 1 patient showed progressive lumbar kyphotic change without aggravation of symptom, and 1 patient showed instrument related infection and underwent removal of screw at 1 year after the surgery.

\section{Surgical outcomes}

The mean operation time was 5.9 hours (range, 5.0-9.5), EBL was $550 \mathrm{ml}$ (range, 300-1,500) with need of average 1.2 packs (range, 0-4) of transfusion, and duration of bed rest was 2.0 days (range, 1-4). The mean length of hospital stay was 26.4 days (range, 8-59), which was longer period than expected, maybe due to long-term antibiotic therapy for infectious patients. Except for 6 infectious patients, mean length of hospital stay was 13.6 days (range, 8-22).

Fortunately, there was no major perioperative morbidity except for a single case of reoperation for resolving hemoperitoneum in patient who underwent anterior corpectomy and fusion. 


\section{DISCUSSION}

The pedicle screw fixation has been used as a universal spinal fusion surgery method for many spinal disorders. Standard open technique for pedicle screw fixation, however, has been associated with several disadvantages. During the open technique, extensive tissue dissection and longtime retraction are inevitable to expose entry points of screw and to provide orientation of lateral to medial for optimal screw trajectory. The excessive retraction of muscle can cause ischemic damage and permanent pathological changes of the muscle ${ }^{7,8,20,21)}$. Already, some authors reported that the degree of damage of muscles and back pain after surgery were proportionate to the size and time of retraction during surgery ${ }^{5,17}$. In addition, extensive dissection of paraspinal muscle can cause excessive blood loss and necrosis of tissue, which can be said to increase the need for transfusion and the chance of postoperative infection $^{20)}$. Such problems cause longer bed rest duration, lengthy hospital stay, and significant $\operatorname{cost}^{22}$. Moreover, some authors have suggested that the open technique can cause adjacent segment degeneration due to extensive dissection of paraspinal muscle or iatrogenic injury of facet joint ${ }^{14,19)}$.

As these problems become important matter, recently, minimally invasive PPF was introduced and developed. PPF uses small muscle splitting approach to allow placement of hardware under fluoroscopic guidance. This technique permits accurate hardware placement while avoiding adverse effects of open technique. Based on this concept, many authors have reported about advantages of single- or two-level PPF such as shorter operative time, less paraspinal muscle damage, less need for postoperative oral analgesics, and lower blood loss than open technique ${ }^{9,13)}$. For multilevel (more than 3 levels) PPF, the longer the length of rod is more difficult to insert, and adjusting alignment of pedicle is difficult. However, recently, with introduction of new instruments, multilevel PPF method that can do a wide range of spinal fusion was developed. Multilevel PPF has usually been carried out for degenerative scoliosis patients1, but it is still not widely carried out yet, and so the clinical result of the operation method are not known well.

The results of the author's present study demonstrated favorable clinical and radiological outcomes. In the current study, with regard to patient's symptom and satisfaction, improvement of clinical outcomes such as VAS and ODI were significant. Also, radiological outcomes including rate of screw malposition (4.1\%), fusion rate (88.2\%), occurrence of definite change of ASA (11.8\%), and occurrence of screw looseing (11.8 $\%)$ were satisfactory. Although it is difficult to compare our results with the other studies because there are many factors affecting outcomes, such as the patient's medical condition, surgeon's experience, surgical indication, surgical method, and outcome assessment criteria, the outcomes in the present study are comparable to other reports ${ }^{11,23)}$.

Multilevel lumbar fusion surgery is needed for multilevel decompression and spinal stability. However, in some special cases, the choice of multilevel fusion is cautious. For example, elderly patients may be at increased risk of surgical morbidities such as pneumonia, cardiovascular event, or wound infection. Also, the patients with medical comorbidities such as cardiovascular disease, renal disease, and diabetes may represent a significant challenge for surgery. The open technique for multilevel fusion, which has the disadvantage of increased operative time and blood loss, may not be tolerated in these elderly patients with poor preoperative general conditions. However, multilevel PPF can be tolerable in even these patients. Compared to the literature ${ }^{6,16}$, based on surgical outcomes of current study, we can demonstrate multilevel PPF to be technically feasible, to be accomplished within tolerable operative times, to be associated with less blood loss than the open technique, and to be associated with short hospital stays. Moreover, there was no surgical complication except for a single case of reoperation due to hemoperitoneum.

Despite all the above, limitations of multilevel PPF have been described, including steep learning curves and theoretically increased radiation exposure ${ }^{3)}$. Also, in rare cases, the inserted instrument comes to be positioned close to skin, which can cause serious skin stimulating symptom, and so instrument removal is needed ${ }^{15)}$.

With development of various instruments of multilevel PPF, it is possible to insert contoured long rod as required for the spinal curvature, and to correct malalignment by compression or distraction. However, the indication is more limited than open technique. In cases of the significant malalignment of pedicle before the surgery due to severe spondylolisthesis (Grade II or more), severe scoliosis, or rotational deformity, it is difficult to carry out reduction of spinal curvature with percutaneous method. Also, since this surgery is carried out under C-arm fluoroscope, the pedicle should be within the range of anatomical structure that can be predicted under fluoroscope by the performing physician. For example, in cases of excessive degeneration and formation of osteophyte, it is hard to find pedicle under fluoroscope, and so accurate test is necessary and attention should be paid before the surgery. Moreover, as the shape of rod which is inserted percutaneously is limited in lordotic or mild kyphotic curved form, it is difficult to apply for severe kyphotic deformity. So, as mentioned above, patients with excessive spondylolisthesis or severe spinal deformity were excluded from this multilevel PPF.

There are some limitations to the present study that should 
be dealt with. This study was retrospective and had a small patient group with too short follow-up period. Furthermore, the study population is heterogeneous with varying indications for spinal fixation, and there is no comparison with open techniques. Additional study is required to compare the multilevel PPF to the open technique in cases of identical operative indications.

\section{CONCLUSION}

Using newer posterior percutaneous instruments, it is possible to achieve multilevel PPF for various lumbar spinal diseases, with favorable clinical, radiological, and surgical outcomes. It remains to be seen whether long-term outcomes are also favorable. Also, the preoperative pedicle alignment is an important factor in multilevel PPF.

\section{REFERENCES}

1. Anand N, Baron EM, Thaiyananthan G, Khalsa K, Goldstein TB: Minimally invasive multilevel percutaneous correction and fusion for adult lumbar degenerative scoliosis: a technique and feasibility study. J Spinal Disord Tech 21:459-467, 2008

2. Brantigan JW, Steffee $\mathrm{AD}$ : A carbon fiber implant to aid interbody lumbar fusion: Two-year clinical results in the first 26 patients. Spine 18:2106-2117, 1993

3. Eck JC, Hodges S, Humphreys SC: Minimally invasive lumbar spinal fusion. J Am Acad Orthop Surg 15:321-329, 2007

4. Foley KT, Gupta SK: Percutaneous pedicle screw-rod fixation of the lumbar spine: preliminary clinical results. J Neurosurg 97: 7-12, 2002

5. Gejo R, Matsui H, Kawaguchi Y, Ishihara H, Tsuji H: Serial changes in trunk muscle performance after posterior lumbar surgery. Spine 24:1023-1028, 1999

6. Hu SS: Blood loss in adult spinal surgery. Eur Spine J 13:S3-S5, 2004

7. Kang JS, Lee YK, Cho J, Moon CT, Chang SK: Changes of muscle related enzymes after posterior approaches to the lumbar spine. J Korean Neurosurg Soc 35:23-28, 2004

8. Kawaguchi Y, Matsui $\mathrm{H}$, Tsuji $\mathrm{H}$ : Back muscle injury after posterior lumbar spine surgery. Part 2: Histologic and histochemical analyses in humans. Spine 19:2598-2602, 1994

9. Kim DY, Lee SH, Chung SK, Lee HY: Comparison of multifidus muscle atrophy and trunk extension muscle strength: percutaneous versus open pedicle screw fixation. Spine 30:123-129, 2005
10. Kim JS, Choi WG, Lee SH: Minimally invasive anterior lumbar interbody fusion followed by percutaneous pedicle screw fixation for isthmic spondylolisthesis: minimum 5-year follow-up. Spine J 10:404-409, 2010

11. Kim NH, Lee JW: Anterior interbody fusion versus posterolateral fusion with transpedicular fixation for isthmic spondylolisthesis in adults. A comparison of clinical results. Spine 24: 812-816, 1999

12. Lee JH, Lee SG, Park CW, Kim WK: Clinical outcome of twolevel percutaneous pedicle screw fixation in lumbar degenerative disease: A preliminary report. Korean J Spine 7:137-142, 2010

13. Lee SH, Choi WG, Lim SR, Kang HY, Shin SW: Minimally invasive anterior lumbar interbody fusion followed by percutaneous pedicle screw fixation for isthmic spondylolisthesis. Spine J 4:644-649, 2004

14. Lim TG, Lee SG, Park CW, Kim WK: Comparative analysis of clinical outcomes and adjacent level angle change on singlelevel lumbar 4-5 fusion using percutaneous screw fixation and open screw fixation. Korean J Spine 7:228-233, 2010

15. Lowery GL, Kulkarni SS: Posterior percutaneous spine instrumentation. Eur Spine J 9:S126-S130, 2000

16. Möller $\mathrm{H}$, Hedlund R: Instrumented and noninstrumented posterolateral fusion in adult spondylolisthesis-a prospective randomized study: part 2. Spine 25:1716-1721, 2000

17. Rantanen J, Hurme M, Falck B, Alaranta H, Nykvist F, Lehto $\mathrm{M}$, et al: The lumbar multifidus muscle five years after surgery for a lumbar intervertebral disc herniation. Spine 18:568-574, 1993

18. Ringel F, Stoffel M, Stüer C, Meyer B: Minimally invasive transmuscular pedicle screw fixation of the thoracic and lumbar spine. Neurosurgery 59:361-366, 2006

19. Schlegel JD, Smith JA, Schleusener RL: Lumbar motion segment pathology adjacent to thoracolumbar, lumbar, and lumbosacral fusions. Spine 21:970-981, 1996

20. Sihvonen T, Herno A, Palj?rvi L, Airaksinen O, Partanen J, Tapaninaho A: Local denervation atrophy of paraspinal muscles in postoperative failed back syndrome. Spine 18:575-581, 1993

21. Styf JR, Willen J: The effects of external compression by three different retreactors on pressure in the erector spine muscles during and after posterior lumbar spine surgery in humans. Spine 23:354-358, 1998

22. Thomsen K, Christensen FB, Eiskjaer SP, Hansen ES, Fruensgaard S, Bünger CE: 1997 Volvo Award winner in clinical studies. The effect of pedicle screw instrumentation on functional outcome and fusion rates in posterolateral lumbar spinal fusion: a prospective, randomized clinical study. Spine 22:2813-2822, 1997

23. Zhao J, Hou T, Wang X, Ma S: Posterior lumbar interbody fusion using one diagonal fusion cage with transpedicular screw/rod fixation. Eur Spine J 12:173-177, 2003 